\section{The anorexia of aging: impact on health and quality of life}

\author{
Vincenzo Di Francesco, Luca Pellizzari, \\ Luigi Corrà, Giorgia Fontana
}

Geriatric Unit A, Azienda Ospedaliera Universitaria Integrata di Verona, Verona, Italy

\begin{abstract}
Malnutrition for the elderly is a serious threat to their health. An elderly malnourished person is more exposed to illness, to disability and to institutionalization. Among the causes of malnutrition there is also the reduction of food intake for reduced hunger. With aging many factors favor the prevalence of satiety with respect to the desire to eat. This condition has been defined as anorexia of aging. Gastric motility impairment, unbalanced central and gastrointestinal hormone and peptide dynamics, together with sensory deterioration, depression, social and iatrogenic factors may be found frequently among older person and cause anorexia. Reduced appetite and exaggerated feeling of satiety are also causes of deterioration of quality of life, due to the decrease in the pleasure of eating and the presence of unpleasant, early and prolonged sense of fullness. Understanding the mechanisms that facilitate anorexia could help eliminate reversible causes and reduce the effect of para-physiological changes at the basis of anorexia. With this approach, it would be possible to improve the nutritional status and, at the same time, the level of quality of life of aged people.
\end{abstract}

\section{Introduction}

Malnutrition is a frequent condition in the elderly, it is associated with a reduction in the adaptive response to the physiological and pathological conditions of aging. ${ }^{1}$ This condition has a dramatic impact on health in the elderly: it impairs the immune system, thus increasing the frequency and severity of infectious diseases. ${ }^{2}$ Malnutrition may cause anemia as well as cognitive decline, osteopenia, altered drug metabolism and sarcopenia. Malnourished elderly run a higher risk of hospitalization, delayed discharge from the hospital and mortality. ${ }^{3}$ Unfortunately, malnutrition is a frequent condition at older ages. It has been reported that $17-65 \%$ of hospitalized older subjects and $5-59 \%$ of institutionalized suffer from malnutrition. ${ }^{4}$

A low food intake represents one of the main risk factors for malnutrition: this condition has been referred to as anorexia of aging. ${ }^{5-8}$ Both cross-sectional ${ }^{9}$ and longitudinal ${ }^{10,11}$ observations have shown that energy intake is reduced in the elderly as compared to younger subjects. The mean calorie reduction between 20 and 80 years of age was $1300 \mathrm{kcal} /$ day for men and 600 for women, ${ }^{9}$ while from 40 to 70 years of age food intake was reduced by $25 \% .^{10}$ At more advanced ages, energy intake declined constantly by 13 $\mathrm{kcal} /$ day/year in women and 25 in men. ${ }^{11}$ The consistent reduction of food intake in the elderly is only in part justified by reductions in energy expenditure, so old men and women mainly lose body weight. ${ }^{11}$

The impact of anorexia not only involves nutritional status and thus healthy, but also quality of life (QoL) at advanced ages. Reduction of food desire and eating pleasure attenuation may themselves cause worry, sadness and ultimately worsening of QoL, regardless the nutritional balance. ${ }^{12}$

The purpose of this manuscript is to review the recent literature by Pubmed search looking for the impact of anorexia on health and quality of life in the elderly. Aim of the study is to describe what are the clinical features and diagnostic tools that allow identification. Finally, this review will try to clarify the pathogenic mechanisms of anorexia and the possible interventions for reducing the consequences of anorexia on nutritional status and on the quality of life of older people.

\section{Prevalence}

In a meta-analysis on 59 international trials, authors from South Australia verified the effect of aging on appetite and energy intake, after overnight fasting and in a postprandial state. They found that energy intake was 16\%-20\% lower in older than younger adults. Hunger was $25 \%$ lower in fasting condition, $39 \%$ lower in a postprandial state. Fullness was $37 \%$ greater in older than younger adults. ${ }^{13}$

In one multicenter study conducted in Italy, authors investigated the prevalence of anorexia in a cohort of about five hundred people aged more than 65 years living in the community, in nursing homes, and in rehabilitation and acute wards. ${ }^{14}$ In this study, anorexia was defined as a reduction in food intake, equal to or greater than $50 \%$ of the Italian recommended daily allowance over a 3-day period, and not attributable to dysphagia or oral pain. The prevalence of
Correspondence: Vincenzo Di Francesco, Geriatric Unit A, AOUI Verona, Piazzale Stefani 1, 37126 Verona, Italy. Tel.:

+39.045.8123218 - Fax: +39.045.8122680.

E-mail: vincenzo.difrancesco@aovr.veneto.it

Key words: Anorexia; aging; malnutrition; quality of life.

Contributions: each of the undersigned authors contributed to the manuscript with the design of the study, the collection of articles and the revision of the manuscript; VDF wrote the paper and drew figure and tables.

Conflict of interest: the authors declare no potential conflict of interest.

Received for publication: 2 February 2018.

Revision received: 19 March 2018.

Accepted for publication: 1 June 2018.

This work is licensed under a Creative Commons Attribution-NonCommercial 4.0 International License (CC BY-NC 4.0).

(C) Copyright V. Di Francesco et al., 2018

Licensee PAGEPress, Italy

Geriatric Care 2018; 4:7324

doi:10.4081/gc.2018.7324

anorexia was reported to be approximately $30 \%$ in the rehabilitation and acute wards and nursing homes. Approximately $11 \%$ of men and $3 \%$ of women in the community were ingesting too few calories.

In another cross-sectional study of 1247 people aged 60 years and older, in Mexico City, anorexia of aging was considered when a person reported eating less in the previous 12 months because of a loss of appetite. Anorexia of aging was reported to be present in $30 \%$ of the study population, and associated with reduced mobility and disability. ${ }^{15}$

\section{Screening and diagnostic tools}

Early detection and treatment of anorexia may prevent its consequences on nutritional status, health and QoL. There are simple, validated clinical tools that objectively quantify appetite in older adults at risk of weight loss. Several groups use nutritional risk assessment tools such as the Mini Nutritional Assessment tool (MNA), the short-form of the MNA (MNA-SF), the Seniors in the Community Risk Evaluation for Eating and Nutrition tool (SCREEN), and the Functional Assessment of Anorexia Cachexia Therapy (FAACT) questionnaire. However, most of these tools are time-consuming and evaluate multiple interdepend- 
ent nutritional domains, but not specifically anorexia. $^{8}$

A more specific simply screening tool has been developed by shortening the 8item Council on Nutrition appetite questionnaire (CNAQ). This 4-item derivative, the Simplified Nutritional Appetite Questionnaire (SNAQ), is a short, simple appetite assessment tool that predicts weight loss in community-dwelling adults and long-term care residents. ${ }^{16}$ More recently SNAQ was utilized on hospitalized older women and could recruit $42 \%$ of patients with a lower score, who showed worse health outcomes at 6-months follow-up. ${ }^{17}$

Off course screening test for anorexia have a clinical relevance only if included in a multidimensional geriatric assessment.

\section{Causes of secondary anorexia}

Many of the social, psychological and organic conditions that characterize aging are also recognized causes of reduced food intake and malnutrition. Among social factors, economic problems and isolation are the most frequent. Depression more frequently plays a role in weight loss in the elderly than in young people. Dementia causes malnutrition, as meals are frequently ignored or refused. Ageusia of swallowing may also be recognized, particularly in post ischemic dementia.

Several diseases, which are frequently associated with the elderly lead to weight loss, mainly by elevating energy expenditure, but also because they depress hunger sensation. Patients with chronic obstructive pulmonary disease suffer from both the consequences of energy dispersion (due to respiratory inefficiency) and anorexia, caused by inflammatory mediators. Similarly, neoplasia and heart failure cause cachexia for higher energy requirement but also for anorexia. In these conditions, high concentrations of cytokines such as IL-6 and TNFalpha have a strong anorexigenic effect and the condition is defined cachexia. ${ }^{18}$ Multiborbidity in general is a possible cause of anorexia.

Dysphagia and poor mastication interfere with nutritional status either directly or indirectly, by reducing taste sensation.

Many drugs, which are widely used in the elderly, may cause anorexia. Table 1 shows a list of the most common. The drugs listed are among the most utilized and cannot generally be easily suspended. However, it is important to look for alternatives in patients at risk of malnutrition. When it is not possible to stop treatments, one should consider a nutritional active intervention to balance the reduced desire to eat.

\section{Mechanisms of primary anorexia}

In similar health and social conditions, energy intake has been reported to be lower in the elderly compared to younger adults. A primitive age-related alteration in the mechanisms regulating energy balance has been hypothesized. ${ }^{19}$

Aging is associated with impairment in the ability to regulate body weight. A study from Roberts et al. ${ }^{20}$ demonstrated that after a few weeks of a $30 \%$ calorie restriction, both elderly and young subjects lost weight. However the elderly were unable to spontaneously regain weight by a compensative hyperphagia as was observed in young adults.

Single food-specific sensory satiety contrasts the excessive intake of a single food even in a normocaloric meal. In the elderly this kind of satiety seems to be impaired thus facilitating monotonous single food intake. ${ }^{21}$ Furthermore, a reduction of taste and smell restricts the choice to a few foods in the elderly. These conditions often lead to a poor alimentary variety of choice that increases the risk of quantitative malnutrition due to low-calorie intake and qualitative low intake of single nutrients (in particular proteins). ${ }^{22}$

\section{Hunger-satiety control in normal condition}

Neurons from the hypothalamic nucleus arcuatus (ARC) release NPY (neuropeptide $\mathrm{Y})$, the most potent central orexigenic mediator, and AgRP (agouti related peptide), another hunger mediator. Axons from ARC neurons release NPY and AgRP in the paraventricular nucleus (PVN), referred to as the satiety center, and inhibit it. Other neural terminations from ARC reach the lateral hypothalamic area (LHA), known as the hunger center, and stimulate it. The net effect of NPY and AgRP tone is hunger stimulation and satiety inhibition leading to increased food intake. ${ }^{23}$ Adjacent to orexigenic neurons in the $\mathrm{ARC}$, other neurons express POMC (pro-opiomelacortin), the precursor of melanocortins, such as alpha MSH, that inhibit LHA and stimulate PVN. Other neurons with similar action express CART (cocaine-amphetamine-related peptide). MSH and CART are inhibited by NPY neurons..$^{23}$

Leptin produced by the adipose tissue and insulin from the pancreas act in the blood as a long-term signal of adiposity, thus indicating the presence of a positive energy balance to the hypothalamus. ${ }^{24}$

CCK, Glucagon-Like peptide 1 (GLP1) and peptide YY (PYY) produced after meals by the upper and lower intestine, and the colon respectively, elicit short-term signal of satiety. Leptin, insulin, CCK, GLP-1 and PYY inhibit NPY/AgRP neurons and stimulate POMC neurons, causing satiety. ${ }^{23,24}$

Ghrelin, a hormone produced by the empty stomach during fasting, stimulates NPY neurons in the ARC, potentiates hunger and triggers eating. ${ }^{25}$ Along the vagal nerve, stimuli from stretching receptors of the stomach rise after gastric filling by food and reach the nucleus of the tractus solitarius (NTS). NTS evokes the sensation of satiety and contrasts excessive food ingestion. Following the same neural pathway, CCK, produced by the intestine in the presence of lipids and amino acids in the lumen, also stimulates NTS and thus postprandial satiety. NTS receives inhibitory fibers from LHA and excitatory fibers from PVN. In this complex system hunger prevails in fasting conditions, satiety after meals. $^{23,24}$

At the level of the central nervous system, endogenous cannabinoids stimulate hunger since they potentiate the pleasure of and desire to eat. ${ }^{26}$ In analogy with animal studies, endocannabinoid tone in the elderly may be diminished. ${ }^{27}$

Taste and flavor, but also the sight of pleasant food, enhances hunger by acting on

Table 1. Drugs that may impair food intake.

\begin{tabular}{ll}
\hline Cardiovascular & Amiodarone \\
& Furosemide \\
& Digoxin \\
& Spironolactone \\
& Theophylline \\
NCS & Levodopa \\
& Fluoxetine \\
& Lithium \\
& Topiramate \\
& Benzodiazepines \\
\hline Metabolic & Metformin \\
& Gliptins \\
Gastrointestinal & H2-antagonists, PPI \\
& Oils \\
& Psyllium \\
\hline Antibiotics & Metronidazole, Macrolides \\
Anti-inflammatory & Colchicine \\
& AlNSD \\
& Penicillamine \\
\hline Chemotherapies & Any \\
Polipharmacy in general
\end{tabular}


the orexigenic mediators and by cortical integration of past experience.

In summary ghrelin is the only orexigenic signal that comes from peripheral districts; it is produced in a pulsatile manner by the empty stomach. All other peripheral short- and long-term signals inhibit hunger and produce satiety. Anorexigenic signaling from the gastrointestinal tract counteracts food intake over digestive and absorption ability. ${ }^{28}$ Adiposity signals from leptin and insulin indicate to the central system energy storage in the adipose tissue, thus they potentiate central satiety sensation. Longand short-term signals interact so that insulin inhibits ghrelin and leptin enforces the CCK signal and vice versa. ${ }^{23}$

Finally, nutrients themselves influence food intake, in particular blood levels of fatty acids and glucose, directly or indirectly, regulate hunger and satiety control centers. ${ }^{23,29}$

\section{Age-related abnormalities of hunger-satiety balance}

$N P Y$ is one of the most abundant peptides in the whole brain and is able to integrate the metabolic and behavioral systems ${ }^{30}$ pushing towards the search and intake of food, as a survival instinct (Figure 1). NPY impairment may be involved in the pathogenesis of anorexia in the elderly. Most of the data have been obtained from observations in rats. Aged rats exhibited low drinking and eating responses when NPY was injected into the PVN. ${ }^{31}$ However, when plasma and cerebrospinal NPY levels were evaluated in anorectic elderly, high levels of NPY were found. ${ }^{29}$

Although some authors found lower concentrations of plasmatic endocannabinoids in elderly as compared to young people, ${ }^{32}$ the opioid inhibitor naloxone showed the same suppressive action on hunger in both young and older subjects, suggesting that there is no difference in the basal endocannabinoid tone. On the basis of these findings, MacIntosh et al. ${ }^{33}$ concluded that age is not associated with reduced opioid feeding drive.

\section{Taste and smell alterations}

The elderly have a reduced sensor-specific satiety. As a consequence they may consume a large amount of a single food, but, on the other hand, they may feel satiety for any food after a single food ingestion. ${ }^{19}$ The possible mechanism for reduced sensory-specific satiety could be impairment of taste and smell senses in the elderly. This latter condition also causes reduced food intake, since taste and smell normally potentiate the sensation of hunger. ${ }^{34}$

Taste is impaired with aging. Fukunaga et $a l .{ }^{35}$ found higher thresholds for recog- nizing sweet, bitter, salty and acid in the elderly as compared with young controls. Oral taste receptors were not reduced, since taste buds count was similar between the different age groups. However, impaired cell turnover and structural abnormalities have been reported with aging. ${ }^{36}$

Olfactory deficit has been demonstrated in the elderly and this may also reduce hunger. ${ }^{37}$ Food consumption increased when elderly subjects were offered food with flavor enhancement. ${ }^{38}$ Difficulty in recognizing flavors might be the consequence of age-related modification of olfactory epithelium, receptors and neural pathways. Furthermore, several drugs, commonly prescribed to old patients, impair taste and smell sensitivity. ${ }^{34}$

Both flavor and taste of food perception are involved not only in specific food recognition, but also in the pleasantness of food. For this reason age-related sensory impairment may affect the hedonistic enhancement of food intake and contribute to the anorexia of aging. ${ }^{2}$ Unfortunately, instead of improving the taste of food, to make it more appetizing, older people often give up the pleasure of eating and choose a monotonous diet, thus increasing the risk of malnutrition.

\section{Gastrointestinal motility}

Abnormalities in gastrointestinal motility may play a crucial role in the hunger/sati- ety unbalance observed with aging. In particular, delayed gastric emptying may cause prolonged postprandial satiety. ${ }^{39-44}$ Studies on gastric motility in the elderly have shown controversial results, mostly due to differences in test meals and methods of evaluation. Scintigraphic observations with radiolabeled food showed delayed emptying of both liquid and solid components of food, ${ }^{40,43}$ but in one study only liquid food emptying from the stomach was delayed. ${ }^{41}$

Ultrasound studies extrapolate gastric volume modifications, and thus gastric filling and emptying, by measuring the area at the level of the antrum, in fasting conditions and at regular intervals after meals. Using this technique, liquid meal emptying was found to be accelerated in the early postprandial phase in elderly patients. ${ }^{42}$ The authors hypothesized an age-related impaired adaptation of the stomach to the alimentary (liquid) bolus. Morley et al. postulated reduced production of nitric oxide at the level of the fundus of older people, leading to a loss of gastric compliance and more rapid antral filling. ${ }^{45}$ Reduced fundus compliance may reduce food intake because of distension of the antrum due to earlier delivery of food. Under more physiological conditions, subjects were given a mixed solid-liquid meal. Ultrasonographic antral dynamics demonstrated delayed gastric emptying in the elderly. A study conducted in Italy showed complete gastric emptying

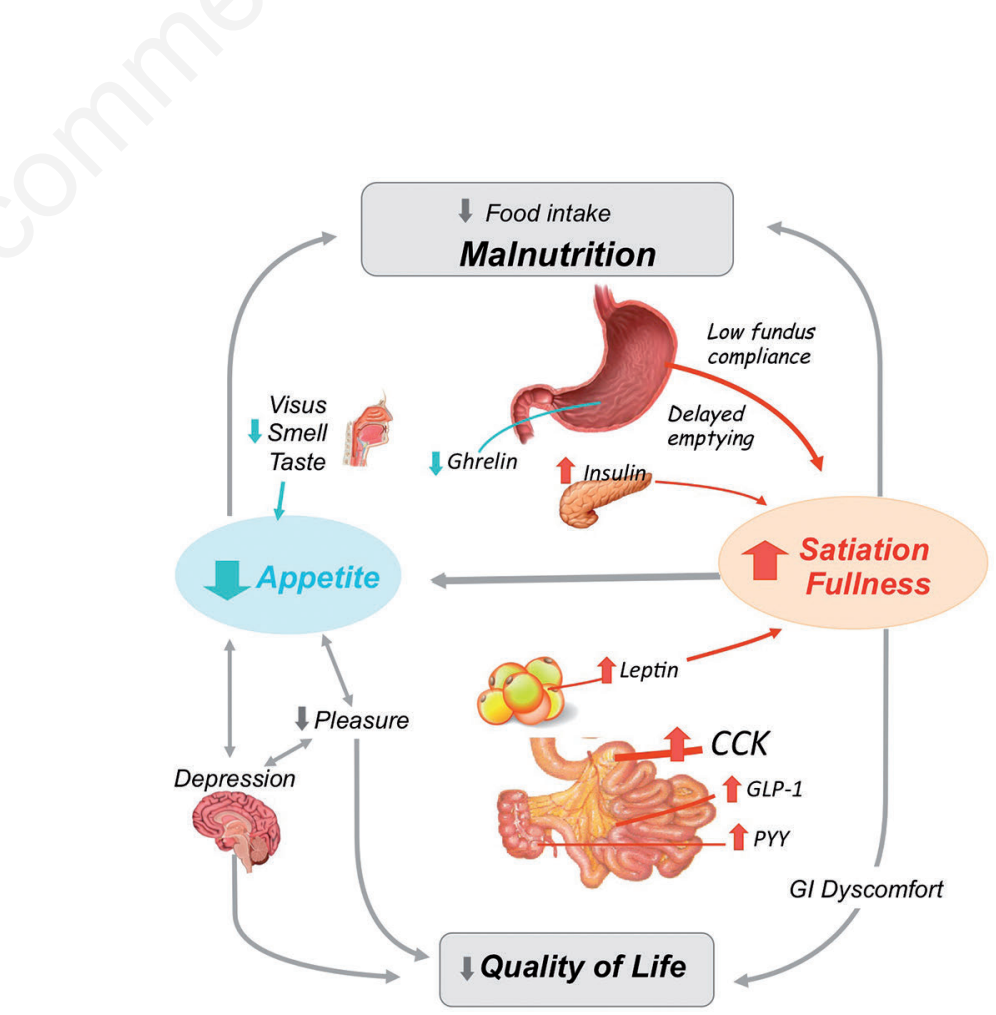

Figure 1. Primary causes and consequences of food intake balance abnormalities in the elderly. CCK, cholecystokinin; GLP-1, glucagon-like peptide 1; PYY, peptide YY. 
after an 800 -kcal meal to be delayed by more than 2 hours in the elderly. ${ }^{39}$ Satiety consistently lasted longer and hunger was suppressed during the 4 hours of postprandial observation; satiety was directly and hunger inversely correlated with gastric emptying time. Nonetheless, satiety remained high in the elderly even at the end of the observation, when only a small quantity of food was present in the antrum. This suggests a possible sensory hypersensitivity. Ultrasonography can only evaluate antral emptying and cannot study fundus filling or emptying, nor distinguish between the liquid and solid components of food. Nevertheless, complete gastric emptying time, extrapolated by ultrasound antral area dynamics, proved to be reliable when compared with gold-standard scintigraphy. ${ }^{46}$ Therefore, the use of ultrasound monitoring after physiological meals is probably the best non-invasive way of evaluating gastric motility at more advanced ages. Furthermore, in physiological conditions, antropyloric motility leads neuro-hormonal response to a meal, which regulates food intake and digestion, according to antral filling and nutrient delivery from the antrum to small intestine. ${ }^{47}$

Slower gastric emptying in elderly persons may be a consequence of a reduced digestive ability of the stomach; it could also be the consequence of an ileal brake effect, caused by a neuro-hormonal response to the presence of nutrients in the small intestine over its digestive and absorptive ability. Finally, chronic gastritis or medications may cause hypochloridria, which further retards gastric emptying. ${ }^{48}$

Abnormalities in gastric motility may cause early satiation due to reduced fundus compliance, and prolonged satiety caused by slower gastric emptying. ${ }^{2}$ Using satiety scores, younger subjects crossed hunger sensation 2 hours after their meal and had scores comparable to fasting values after 4 hours. In their elderly counterparts, the sensation of satiety still prevailed over hunger 4 hours after their meal. Satiety was directly and hunger inversely correlated with gastric emptying time. ${ }^{39}$

Intestinal motility also influences the hunger/satiety balance at the level of colonrectum, in particular colonic stasis delays gastric emptying by a colon-gastric reflex, indirectly prolonging the sensation of satiety. ${ }^{5}$ Constipation, which is a common finding in advanced ages, is an exclusion criteria for motility studies of this kind, but it should be taken into account as a possible cause of impaired motility along the whole gastrointestinal tract, and thus as a risk factor for anorexia. ${ }^{49}$

More recently it has been shown that gut microbiota promotes satiety and lowers hunger and energy intake in humans, by fermentation of prebiotics. ${ }^{50}$ Significant changes in gut microbiota composition in older subjects have been reported in relation to diet, drug use and the place of residence. Moreover, changes in microbiota composition in the old age have been related to immunosenescence and inflammatory processes. ${ }^{51}$ Further studies are needed to clarify a possible role for microbiota on hunger/satiety balance in the Elderly.

\section{Role of hormones}

$C C K$ is the most relevant satiety hormone: it is released by the proximal small intestine in response to the delivery of nutrients from the antrum, particularly of lipids and proteins. ${ }^{28}$ Several studies have demonstrated the presence of higher CCK concentrations in the blood of aged compared to young persons..$^{39,47,52,53}$ Intraduodenal infusion of either glucose solution or lipid suspensions showed a greater difference between the age groups after lipid infusion. ${ }^{54}$ Motor gallbladder sensitivity to CCK seems to be impaired with aging, but a CCK satiating effect sensitivity seems to be preserved. ${ }^{55}$ High levels of CCK are among the major causes of the anorexia of aging as they were correlated to abnormally higher satiety sensation after meals. ${ }^{47}$ In a study we confirmed higher fasting and postprandial CCK serum concentration in the elderly. ${ }^{39}$ At the end of the observation, when the antrum contained only small volumes of food, the elderly group still had high circulating CCK levels, high satiety and low hunger.

CCK may have a primitive role in the genesis of anorexia and malnutrition, since higher CCK levels were found in malnourished aged subjects compared to well-nourished elderly. ${ }^{53}$

Peptide YY (PYY) is released by the distal intestine in response to the presence of undigested nutrients in the lumen. PYY inhibits the NPY-mediated appetite stimulus. ${ }^{55} \mathrm{~A}$ previous study on postprandial PYY did not find any differences between young and older people, but the observations were limited to $120 \mathrm{~min}$ after the meal. ${ }^{47}$ A more recent observation showed a greater rise of PYY in late postprandial period in the elderly as compared to young controls. ${ }^{39}$ In analogy with CCK, abnormally high postprandial PYY levels may inhibit the search for a second meal, thus leading to longer fasting intervals, causing reduced food intake and malnutrition in elderly persons. Both CCK and PYY are enteric peptides involved in gastrointestinal motility in response to eating, they provide a potent anorexigenic signals to the hypothalamus. They also mediate slowing of gastric emp- tying induced by the presence of nutrients in the small intestine. ${ }^{47}$ Abnormally high levels of fasting and postprandial CCK and PYY may further and indirectly prolong satiety by slowing antral emptying.

The GLP-1 is an active by-product from glucagon metabolism. GLP-1 is produced by the distal small intestine after food ingestion and elicits an anorexigenic signal. ${ }^{56}$ The role of GLP-1 in anorexia in the elderly needs to be further investigated since its concentrations after different stimuli have been shown to be similar in older and younger subjects. ${ }^{47,53}$ However after a hyperlipidic meal, but not after carbohydrates load, GLP-1 curves and satiety sensation were higher in older group compared to young controls. ${ }^{57}$

Insulin, the well-known regulator of glucose metabolism, is also a satiety hormone. Insulin probably acts indirectly by enhancing the leptin signal to the hypothalamus and by inhibiting the ghrelin orexigenic stimulus. Aging is characterized by reduced glucose tolerance and elevated insulinemia. This condition may facilitate anorexia. Higher concentrations of plasma insulin may amplify the anorexigenic signal of leptin, since insulin stimulates central leptin action and sensitivity at the level of the ARC. ${ }^{58}$ Hyperinsulinemia in the elderly could also be responsible for inhibiting ghrelin gastric expression and central sensitivity. ${ }^{59}$

Leptin is another possible actor of anorexia in the elderly. This cytokine is a hormone that is mainly produced by adipose cells, whose main role consists in long-term energy balance, by giving the CNS a sign of energy storage. As discussed before, low leptin levels signal loss of body fat and a need for energy intake, while high leptin levels testify the presence of adequate body fat and no need for further food intake. $^{23}$

Fasting leptin in healthy elderly subjects was found to be elevated when compared to young persons, even after adjusting for body fat mass. ${ }^{60}$ Serum leptin was found to be significantly higher in the elderly group after a meal. Interaction was not significant, as well as the effect of time, so that mean values of leptin showed a flat line in both young and older persons; younger subjects showed lower mean values compared to older subjects. ${ }^{61}$ These findings are in line with previous observations in adults, suggesting that leptin concentrations do not change significantly shortly after a meal ${ }^{62}$ and confirming that leptin is more involved in long-term food control than in short-term modulation of food intake.

Nevertheless, the authors hypothesized that elevated serum leptin may have facilitated a postprandial prevalence of anorexi- 
genic signals. As already reported, aging is characterized by high postprandial CCK concentrations. ${ }^{5,39}$ In this condition, leptin passes more easily through the blood-brain barrier, inducing an increase in hypothalamic sensitivity to leptin. ${ }^{63}$ Other authors claim the contrary for higher central CCK sensitivity in the presence of high leptin levels. ${ }^{62}$ In any case, leptin and CCK collaborate to amplify the inhibitory message to NPY feeding drive.

Ghrelin is the only peripheral hormone known to stimulate appetite. Ghrelin is produced and secreted by the endocrine gastric mucosa to enhance food intake. ${ }^{25}$ Ghrelin has a pulsatile secretion, with a maximal blood concentration just before the meal and a drop just after stomach filling. For these reasons, ghrelin is thought to be implicated in meal initiation. It has been suggested that ghrelin also encourages healing and growing. Therefore, hunger elicited by ghrelin could be considered as a warranty for energy surplus need in case of growth and tissue repair. ${ }^{23-25}$

Ghrelin also seems to regulate longterm energy balance, and it rises under conditions causing negative balance such as anorexia nervosa, cachexia or hypocaloric diet. On the contrary, ghrelin secretion is suppressed in obese subjects with a positive energy balance. ${ }^{64}$

Only relatively few data are available on ghrelin dynamics with aging. One study showed elevated fasting ghrelin values in the elderly. ${ }^{65}$ Two other observations showed no significant age-related difference even in the postprandial period. ${ }^{53,61}$ Postprandial ghrelin was not significantly different in young and old subjects. In both age groups ghrelin dropped after the meal and returned to basal values within $4 \mathrm{~h}$. Nevertheless, hunger did not follow a postprandial ghrelin raise in the elderly. Concurrent high concentrations of leptin and insulin may have been responsible for the low sensitivity to ghrelin..$^{59,64}$ Furthermore, ghrelin is produced by the stomach in two major molecular forms: an active acylated ghrelin that stimulates food intake, and a second desacyl ghrelin that was thought to have no hormonal action. ${ }^{66}$ However, in animal models, it has been demonstrated that, in contrast with the acylated form, desacyl ghrelin decreases the intake of food. ${ }^{64}$ The ratio of acylated to non-acylated ghrelin may have a role in different responses to ghrelinemia in the elderly. In fact on a small sample of healthy subjects lower fasting and postprandial plasmatic acylated ghrelin was found in the older subjects, without the pulsatile response demonstrated in younger controls after the meal. ${ }^{67}$

\section{Intervention}

In summary, the risk of malnutrition in the elderly is high for many clinical, functional and social risk factors. A primitive socalled anorexia of aging represents an additional primary risk factor. In adult life we face the risk of overeating and gaining body weight due to the prevalence of hunger (mostly central) signals, warranting survival in the presence of small quantities of food. In the elderly there is a prevalence of both short- and long-term satiety signals (mostly peripheral), which contrast energy balance and cause malnutrition and frailty. ${ }^{68}$ In this case it seems that species survival prevails and less active (aged) people spontaneously consume less community food resources. Notwithstanding the easy and inexpensive access to food in Western countries, these mechanisms lead to malnutrition, frailty, and thus to higher morbidity, disability and mortality at older ages. Even the quality of life is deteriorated by reduction of the hedonistic component of eating and the pleasure of social interactions related to food consumption. So the treatment goal should be the improvement of malnutrition and quality of life, the intervention should increase both the caloric intake and the pleasure of eating.

A pharmacological approach to this multifactorial problem does not seem to be realistic, but a strategy to counteract anorexia and malnutrition can be put in place, with a multidimensional approach that should be aimed at countering the reversible causes of anorexia. Most of these conditions can be contrasted with dietary, environmental and social interventions (Table 2). Even the consequences of physiologic age-related alterations, causing primary anorexia, can be reduced. For example, slow gastric emptying may be contrasted by fractionating food intake into small digestible meals. Improving taste and flavor of the dishes may enhance blunted hunger. Limiting the intake of CCK-stimulating foods, such as fats and proteins, may reduce excessive satiation CCK signal (Table 3). ${ }^{69,70}$

The Literature has not yet clarified all of the aspects of anorexia in the elderly. Many more peptides and mediators involved in energy homeostasis need to be tested in the

Table 2. Reversible conditions causing anorexia and possible intervention.

\begin{tabular}{ll} 
Condition & Intervention \\
Isolation & Social support (relatives, volunteers, health workers) \\
Poverty & Social intervention \\
\hline Distracting meal environment & Modify environment \\
Improper meal time & Adapt the daily meal times to the patient's needs \\
\hline Depression & Psychological support, anti-depressant drugs \\
Edentulia & Diet modification, dental prostheses \\
\hline Oropharingeal mycosis & Anti-fungal treatment \\
Chronic pain & Pain medications and other non-pharmacological analgesic interventions \\
\hline Constipation & Diet modification (water and fibers), medications, toilet training \\
Drugs & Reconsider the indication and the choice of alternative drugs \\
\hline Malnutrition & Calories and proteins supplement \\
Dehydration & Encourage fluid intake between meals \\
\hline Inflammation & Diet modification (antioxidants, fresh fruit, vegetables), supplements \\
Subclinical infection & Diagnose and treat it \\
\hline
\end{tabular}


Table 3. Physiologic causes of anorexia, consequences and possible intervention.

\begin{tabular}{|c|c|c|}
\hline Factors & Effects & Strategy of intervention \\
\hline Diminished sense of smell and taste & $\begin{array}{l}\text { Decreased oral intake, renunciation of flavors } \\
\text { and aromas }\end{array}$ & Propose colorful, fragrant, tasty foods \\
\hline Reduced sensory specific satiety & More monotonous diet & Propose a varied diet \\
\hline Increased cytokines activity & Reduced food intake, catabolism, cachexia & Propose a diet rich in antioxidants; Supplements \\
\hline $\begin{array}{l}\text { Delayed gastric emptying, } \\
\text { reduced gastric compliance }\end{array}$ & Increased satiation and satiety & Provide small, frequent and easily digestible meals \\
\hline Decreased opioid activity & Reduce eating pleasure, reduced oral intake & Support the social and cultural pleasure of eating \\
\hline NPY: Possible decrease with aging & Reduced hunger & Stimulate eating desire \\
\hline Increased CCK & $\begin{array}{l}\text { Increased satiating effect } \\
\text { (which stimulate CCK secretion) }\end{array}$ & Avoid large meal and protein and lipid overload \\
\hline Reduced Ghrelin & Reduced hunger, search for food, meal initiation & Try alternative stimuli (exogenous ghrelin administration?) \\
\hline Increased GLP-1 and PYY & Postprandial fullness & Provide small and easily digestible meals \\
\hline Increased Leptin & Reduced hunger, amplified satiation signals & Encourage exercise (it reduces the leptinic signal) \\
\hline
\end{tabular}

older population, the complex integration of peripheral and central circuits needs to be further studied in humans. Little attention has been paid to the role of digestive and absorptive dysfunction on hunger and satiety control with aging. From basic research it is now time to boost nutritional and multidimensional intervention protocols.

\section{References}

1. Mowé M, Bohmer T, Kindt E. Reduced nutritional status in an elderly population is probable before disease and possibly contributes to the development of disease. Am J Clin Nutr 1994;59:317-24.

2. Thomas DR, Zdrowski CD, Wilson $\mathrm{MM}$, et al. Malnutrition in subacute care. Am J Clin Nutr 2002;75:308-13.

3. O'Shea E, Trawley S, Manning E, et al. Malnutrition in Hospitalized Older Adults: A Multicenter Observational Study of Prevalence, Associations and Outcomes. J Nutr Health Aging 2017; 21:830-6.

4. Vellas B, Lauque S, Andrieu S, et al. Nutrition assessment in the elderly. Curr Opin Clin Nutr Metab Care 2001;4:5-8.

5. Morley JE. Anorexia of aging: physiologic and pathologic. Am J Clin Nutr 1997;66: 760-73.

6. Chapman IM, MacIntosh CG, Morley JE, Horowitz M. The anorexia of aging. Biogerontology 2002;3:67-71.

7. Di Francesco V, Fantin F, Omizzolo F, et al. The anorexia of aging. Dig Dis 2007;25:129-37.

8. Visvanathan R. Anorexia of aging. Clin Geriatr Med 2015;31:417-27.
9. Briefel RR, McDowell MA, Alaimo K, et al. Total energy intake of the US population: the third National Health and Nutrition Examination Survey, 19881991. Am J Clin Nutr 1995;62:1072S$80 \mathrm{~S}$.

10. Hallfrisch J, Muller D, Drinkwater D, et al. Continuing diet trends in men: the Baltimore Longitudinal Study of Aging (1961-1987). J Gerontol 1990;45: M186-91.

11. Baumgartner RN, Koehler KM, Gallagher D, et al. Epidemiology of sarcopenia among the elderly in New Mexico. Am J Epidemiol 1998;147: 755-63.

12. Morley JE, Silver AJ. Anorexia in the elderly. Neurobiol Aging 1988;9:9-16.

13. Giezenaar C, Chapman I, LuscombeMarsh N, et al. Ageing is associated with decreases in appetite and energy intake-a meta-analysis in healthy adults. Nutrients 2016;8:28.

14. Donini LM, Poggiogalle E, Piredda M, et al. Anorexia and eating patterns in the elderly. PLoS One 2013;8:e63539.

15. Vazquez-Valdez OE, Aguilar-Navarro S, Avila-Funes JA. Association between anorexia and disability in older community-dwelling Mexicans. J Am Ger Soc 2010;58:2044-6.

16. Wilson MM, Thomas DR, Rubenstein LZ, et al. Appetite assessment: simple appetite questionnaire predicts weight loss in community-dwelling adults and nursing home residents. Am J Clin Nutr 2005;82:1074-81.

17. Pilgrim AL, Baylis D, Jameson KA, et al Measuring appetite with the simplified nutritional appetite questionnaire identifies hospitalized older people at risk of worse health outcomes. J Nutr Health Aging 2016;203-7.
18. Yeh SS, Schuster MW. Geriatric cachexia: the role of cytokines. Am J Clin Nutr 1999;70:183-97.

19. Rolls BJ, Dimeo KA, Shide DJ. Agerelated impairments in the regulation of food intake. Am J Clin Nutr 1995;62: 923-31.

20. Roberts SB, Fuss P, Heyman MB, et al. Control of food intake in older men. JAMA 1994;272:1601-6.

21. Rolls B J, McDermott TM. Effects of age on sensory-specific satiety. Am J Clin Nutr 1991;54:988-96.

22. Rolls BJ. Do chemosensory changes influence food intake in the elderly? Physiol Behav 1999;66:193-7.

23. Morton GJ, Cummings DE, Baskin DG, et al. Central nervous system control of food intake and body weight. Nature 2006;443:289-95.

24. Havel PJ. Peripheral signals conveying metabolic information to the brain: short-term and long-term regulation of food intake and energy homeostasis. Exp Biol Med 2001;226:963-77.

25. Van der Lely AJ, Tschop M, Heiman ML, Ghigo E. Biological, physiological, pathophysiological, and pharmacological aspects of ghrelin. Endocr Rev 2004;25:426-57.

26. Drewnowski A, Krahn DD, Demitrack MA, et al. Naloxone, an opiate blocker, reduces the consumption of sweet high fat foods in obese and lean female binge-eaters. Am J Clin Nutr 1995;61: 1206-12.

27. Gambert SR, Garthwaite TL, Pontzer $\mathrm{CH}$, Hagen TC. Age-related changes in central neurons system alpha-endorphins and ACTH. Neuroendocrinology 1980;31:252-5.

28. Strader AD, Woods SC. Gastrointestinal hormones and food intake. 
Gastroenterology 2005;128:175-91.

29. Roberts SB, Rosemberg I. Nutrition and aging: changes in the regulation of energy metabolism with aging. Physiol Rev 2006;86:651-67.

30. Leibowitz SF. Brain neurotransmitters and eating behavior in the elderly. Neurobiol Aging 1988;9:20-2.

31. Blanton CA, Horwitz BA, Blevins JE, et al. Reduced feeding response to neuropeptide $\mathrm{Y}$ in senescent Fischer 344 rats. Am J Physiol 2001;280:R1052-60.

32. Martinez M, Hernanz A, Gomez-Cerezo $\mathrm{J}$, et al. Alterations in plasma and cerebrospinal fluid levels of neuropeptides in idiopathic senile anorexia. Regul Pept 1993;49:109-17.

33. MacIntosh CG, Sheehan J, Davani N, et al. Effects of aging on the opioid modulation of feeding in humans. J Am Geriatr Soc 2001;49:1518-24.

34. Schiffman SS. Taste and smell losses in normal aging and disease. JAMA 1997;278:1357-62.

35. Fukunaga A, Uematsu H, Sugimoto K. Influences of aging on taste perception and oral somatic sensation. J Gerontol A Biol Sci Med Sci 2005;60A:109-13.

36. Schiffman SS. Food recognition by the elderly. J Gerontol 1977;32:586-92.

37. Doty RL, Shaman P, Applebaum SL, et al. Smell identification ability: changes with age. Science 1984;226:1441-3.

38. Schiffman SS, Warwick Z. Flavor enhancement of foods for the elderly can reverse anorexia. Neurobiol Aging 1988;9:24-6.

39. Di Francesco V, Zamboni M, Dioli A, et al. Delayed postprandial gastric emptying and impaired gallbladder contraction together with elevated cholecystokinin and peptide YY serum levels sustain satiety and inhibit hunger in healthy elderly persons. J Gerontol A Biol Sci Med Sci 2005;60:1581-5.

40. Horowitz M, Maddern GJ, Chatterton $\mathrm{BE}$, et al. Changes in gastric emptying rate with age. Clin Sci 1984;67:213-8.

41. Moore JG, Tweedy C, Christian PE, Datz FL. Effect of age on gastric emptying of liquid solid meals in man. Dig Dis Sci 1983;28:430-4.

42. Kupfer RM, Heppell M, Haggith JW, Bateman GL. Gastric emptying and small-bowel transit rate in the elderly. J Am Geriatr Soc 1985;33:340-3.

43. Wegener M, Borsch G, Schaffstein J, et al. Effect of ageing on the gastrointestinal transit of a lactulose-supplemented mixed solid-liquid meal in humans. Digestion 1988;39:40-6.

44. Wedmann B, Schmidt G, Wegener M, et al. Effects of age and gender on fatinduced gallbladder contraction and gastric emptying of a caloric liquid meal: a sonographic study. Am J Gastroenterol 1991;86:1765-70.

45. Morley JE, Kumar VB, Mattammal $\mathrm{MB}$, et al. Inhibition of feeding by LNAME: effects of aging. Eur $J$ Pharmacol 1996:311:15-29.

46. Benini L, Sembenini C, Heading RC, et al. Simultaneous measurement of gastric emptying of a solid meal by ultrasound and by scintigraphy. Am J Gastroenterol 1999;94:2861-5.

47. MacIntosch GC, Andrews JM, Jones $\mathrm{KL}$, et al. Effects of age on concentrations of plasma cholecystokinin, glucagon-like peptide-1 and peptide YY and their relation to appetite and pyloric motility. Am J Clin Nutr 1999;69:9991006.

48. Benini L, Castellani G, Bardelli E, et al. Omeprazole causes delay in gastric emptying of digestible meals. Dig Dis Sci 1996;41:469-74.

49. Stivland T, Camilleri M, Vassallo M, et al. Scintigraphic measurement of regional gut transit in idiopathic constipation. Gastroenterology 1991;101:107-15.

50. Cani PD, Lecourt E, Dewulf EM, et al. Gut microbiota fermentation of prebiotics increases satietogenic and incretin gut peptide production with consequences for appetite sensation and glucose response after a meal. Am J Clin Nutr 2009;90:1236-43.

51. Mello AM, Paroni G, Daragjati J, Pilotto A. Gastrointestinal microbiota and their contribution to healthy aging. Dig Dis 2016;34:194-20.

52. MacIntosh CG, Morley JE, Wishart J, et al. Effects of exogenous cholecystokinin (CCK)-8 on food intake and plasma CCK, leptin and insulin concentrations in older and young adults: evidence for increased CCK activity as a cause of the anorexia of aging. J Clin Endocrinol Metab 2001;86:5830-7.

53. Sturm K, MacIntosh CG, Parker BA, et al. Appetite, food intake, and plasma concentrations of cholecystokinin, ghrelin, and other gastrointestinal hormones in undernourished older women and well-nourished young and older women. J Clin Endocrinol Metab 2003;88:3747-55.

54. MacIntosh CG, Horowitz M, Verhagen $M$, et al. Effects of small intestinal nutrient infusion on appetite, gastrointestinal hormone release, and gastric myoelectrical activity in young and older men. Am J Gastroenterol 2001;96:997-1007.

55. Batterham RL, Cowley MA, Small CJ et al. Gut hormone PYY physiologically inhibits food intake. Nature 2002;
418:650-3.

56. Gutzwiller JP, Goke B, Drewe J, et al. Glucagon-like peptide-1: a potent regulator of food intake in humans. Gut 1999;44:81-6.

57. Di Francesco V, Barazzoni R, Bissoli L, et al. The quantity of meal fat influences the profile of postprandial hormones as well as hunger sensation in healthy elderly people. J Am Med Dir Assoc 2010;11:188-93.

58. Doucet E, St-Pierre S, Alméras N, et al. Quebec Family Study: Fasting insulin levels influence plasma leptin levels independently from the contribution of adiposity: evidence from both a crosssectional and an intervention study. J Clin Endocrinol Metab 2000;85:4231-7.

59. Murdolo G, Lucidi P, Di Loreto C, et al. Insulin is required for prandial ghrelin suppression in humans. Diabetes 2003;52:2923-7.

60. Zamboni M, Zoico E, Fantin F, et al. Relation between leptin and the metabolic syndrome in elderly women. J Gerontol A Biol Sci Med Sci 2004;59:396-400.

61. Di Francesco V, Zamboni M, Zoico E, et al. Unbalanced serum leptin and ghrelin dynamics prolong postprandial satiety and inhibit hunger in healthy elderly: another reason for the 'anorexia of aging'. Am J Clin Nutr 2006;83:1149-52.

62. Romon M, Lebel $\mathrm{P}$, Velly $\mathrm{C}$, et al. Leptin response to carbohydrate or fat meal and association with subsequent satiety and energy intake. Am J Physiol 1999;277:E855-61.

63. Barrachina MD, Martinez V, Wang L, et al. Synergistic interaction between leptin and cholecystokinin to reduce short term food intake in lean mice. Proc Natl Acad Sci U S A 1997;94:10455-60.

64. Asakawa A, Inui A, Fujimiya M, et al. Stomach regulates energy balance via acylated ghrelin and desacyl ghrelin. Gut 2005;54:18-24.

65. Rigamonti AE, Pincelli AI, Corrà $\mathrm{B}$, et al. Plasma ghrelin concentration in elderly subjects: comparison with anorexic and obese patients. J Endocrinol 2002;175:R1-R5.

66. Hosoda H, Kojima M, Matsuo H, Kangawa K. Ghrelin and des-acyl ghrelin: two major forms of rat ghrelin peptide in gastrointestinal tissue. Biochem Biophys Res Commun 2000;279: 909-13.

67. Di Francesco V, Fantin F, Residori L, et al. Effect of age on the dynamics of acylated ghrelin in fasting conditions and in response to a meal. J Am Geriatr Soc 2008;56:1369-70.

68. Morley JE. Anorexia, weight loss, and 
frailty. J Am Med Dir Assoc 2010;11: 225-8.

69. Landi F, Lattanzio F, Dell'Aquila G, et al. Prevalence and potentially reversible factors associated with anorexia among older nursing home residents: results from the ULISSE project. J Am Med Dir Assoc 2013;14:119-24.
70. Landi F, Calvani R, Tosato M, et al. Anorexia of aging: risk factors, consequences, and potential treatments. Nutrients 2016;8:69. 